http://dx.doi.org/10.11646/phytotaxa.175.1.4

\title{
Further insights into the taxonomy of the Silene nocturna species complex (Caryophyllaceae): a systematic survey of the taxa from Sardinia and Corsica
}

\author{
GIANLUIGI BACCHETTA ${ }^{1}$, ANGELINO CARTA², GUILHAN PARADIS ${ }^{3}$, CAROLE PIAZZA ${ }^{4}$, LORENZO \\ PERUZZI ${ }^{2 *}$ \\ ${ }^{1}$ Centro Conservazione Biodiversità (CCB), Dipartimento di Scienze della Vita e dell' Ambiente, Università degli Studi di Cagliari, Viale \\ S. Ignazio da Laconi 11-13, I 09123 Cagliari, Italy \\ ${ }^{2}$ Dipartimento di Biologia, Unità di Botanica, Università di Pisa, Via Luca Ghini 13, I 56126, Pisa, Italy; e-mail lorenzo.peruzzi@unipi.it \\ ${ }^{3}$ cours Général Leclerc, F 20000, Ajaccio \\ ${ }^{4}$ Conservatoire botanique national de Corse, Office de l'Environnement de la Corse, Avenue Jean Nicoli, F 20250 Corte \\ *Author for correspondence
}

\begin{abstract}
The taxonomy of Silene nocturna subsp. boullui (putatively endemic to W Corsica) and S. valsecchiae (putatively endemic to E Sardinia) is investigated, by means of morphometric and karyological analyses. Both taxa resulted diploid with $2 \mathrm{n}=24$ chromosomes. On the basis of morphological results, we confirm the species rank for $S$. valsecchiae, and consider $S$. nocturna subsp. boullui as the priority name, at subspecies rank, for $S$. nocturna subsp. capraria (=S. reflexa).
\end{abstract}

Key words: Central Mediterranean, chromosome numbers, morphology, seeds, SEM, Silene sect. Scorpioideae

\section{Introduction}

Recent work on the Silene nocturna Linnaeus (1753: 416) complex in the Central Mediterranean area (Peruzzi \& Carta 2013, Peruzzi et al. 2014a) showed the existence of three taxonomic units: S. mutabilis Linnaeus (1756: 16) [= S. neglecta Tenore (1826: 13)], S. nocturna subsp. nocturna and S. nocturna subsp. capraria (Sommier 1896: 113) Peruzzi \& Carta (2013: 46) [=S. reflexa (Linnaeus 1753: 416) Aiton (1811: 86)]. However, as already pointed out by Peruzzi \& Carta (2013), two further taxa from the Corso-Sardinian biogeographic province need investigation: $S$. nocturna subsp. boullui (Jordan ex Rouy \& Foucaud 1896: 114) Gamisans in Gamisans \& Jeanmonod (1993: 243), originally described from Corsica (see also Jeanmonod 2007, 2013), and S. valsecchiae Bocchieri (1988: 305), putative endemic to eastern Sardinia (Bacchetta et al. 2006a, 2006b, Arrigoni 2010, Pinna et al. 2013).

This paper is part of a broader project on the taxonomic study of the endemic Italian flora (Domina et al. 2012, Peruzzi et al. 2014b), which also led to several recent other publications on the genus Silene (Brullo et al. 2012, 2014, Peruzzi \& Carta 2013, Peruzzi et al. 2014a). The aim of the present study is to provide data concerning morphology, seed micromorphology and karyology of these two taxa, in order to contribute to their correct taxonomic circumscription.

\section{Material and Methods}

Quantitative morphological investigations is performed on herbarium specimens of S. nocturna subsp. boullui and $S$. valsecchiae. The herbaria checked were CAG and PI (codes according to Thiers 2014). The following eight characters were examined: plant size $(\mathrm{cm})$, flower number, inclination of the lowest flower's pedicel [from $0^{\circ}$ (erect and adpressed to peduncle upwards), to $180^{\circ}$ (completely reflexed)], length of the lowest flower's pedicel (mm), calyx length (mm), carpophore length $(\mathrm{mm})$, calyx teeth length and width $(\mathrm{mm})$. 
Silene nocturna Linnaeus (1753: 416) subsp. nocturna

Type (lectotype designated by Ghafoor 1978: 91):-PENNSYLVANIA. [Silene] nocturna 4 HU [Horto Upsaliensis] [on verso:] e semine D. Kalmii ex America, Herb. Linn. No. 583.8 (LINN!).

Specimina visa (besides those listed in Peruzzi \& Carta, 2013):-FRANCE. Corsica: Grotta di u banditu (Farinole), 12 June 2013, C. Piazza s.n. (PI!, three stheets).

Silene nocturna L. subsp. boullui (Jord. ex Rouy \& Foucaud) Gamisans in Gamisans \& Jeanmonod (1993: 243)

Bas.:- Silene mirabilis Rouy \& Foucaud (1896: 114) subsp. boullui Jord. ex Rouy \& Foucaud (1896: 114)

Type:- not designated. Described from "Corse: Rogliano; Bonifacio à la Trinité (Revelière); îles Sanguinaires et la Parata près Ajaccio

(Boullu in herb. Rouy).”. In Herbarium Rouy at LY no specimens which could be considered as original material for this name are conserved (M. Thiébaut, pers. comm.).

= Cucubalus reflexus Linnaeus (1753: 416)

Type (lectotype designated by Talavera in Cafferty \& Jarvis, 2004: 1051):- - Herb. Linn. No. 582.22 (LINN!)

$\equiv$ Silene reflexa $(\mathrm{L}$.$) Aiton (1811: 86)$

= Silene capraria Sommier (1898: 113) ESilene nocturna subsp. capraria (Sommier) Peruzzi \& Carta (2013: 46), syn. nov.

Type (lectotype designated by Foggi et al. 2001: 13):--ITALY. Tuscany: Insula Capraria (Capraja), Punta del Trattoio, 6 April 1896, Sommier s.n. (FI!).

Specimina visa (besides those listed in Peruzzi \& Carta, 2013):- FRANCE. Corsica: Mezzu Mare (Iles Sanguinaires), Ajaccio, 7 May 2013, G. Paradis s.n. (PI!, five sheets).

Silene valsecchiae Bocchieri (1988: 305)

Type:-ITALY. Sardinia: Isola Serpentara, a SE della casa diruta, 11 May 1986, E. Bocchieri s.n. (holotype CAG!).

Specimina visa:--ITALY. Sardinia: Isola dei Cavoli, Cala Is Cascias, 15 May 2005, R. Angius, G. Bacchetta et L. Podda s.n. (CAG!); Isola Serpentara, settore sud-orientale, 7 April 2006, G. Bacchetta, G. Fenu, G. Iiriti et L. Podda s.n. (CAG!, two sheets); Isola di Serpentara, settore centrale, 13 May 2013, G. Bacchetta s.n. (CAG!, three sheets).

\section{Acknowledgements}

Many thanks are due to Francesca Lentini for helping in laboratory work and to M. Thiébaut for information on Silene specimens in LY. Financial funding of University of Pisa (Fondi di Ateneo EX60\%) is gratefully acknowledged.

\section{References}

Aiton, W.T. (1811) Hortus Kewensis Ed. 2, 3. Longman et al., London, 432 pp.

Arrigoni, P.V. (2010) Flora dell'Isola di Sardegna 2. Carlo Delfino Editore, Sassari, 623 pp.

Bacchetta, G., Fenu, G., Iiriti, G., Mattana, E., Meloni, F., Mulè, P. \& Podda, L. (2006a) Territory defence throughout conservation of the plant diversity: the project of the Protected Sea Area of Capo Carbonara (South eastern Sardinia). I International Symposium Environment, Identities and Mediterranean Area Article number 4150533: 302-307.

Bacchetta, G., Fenu, G., Mattana, E. \& Mulè, P. (2006b) Monitoraggio e conservazione della flora e della vegetazione costiera nell'Area Marina Protetta di Capo Carbonara (Sardegna sud-orientale). Atti Simposio su Monitoraggio Costiero Mediterraneo 97-106.

Bocchieri, E. (1988) Silene valsecchiae e Ferula arrigonii, due nuove specie della Sardegna. Bollettino della Società Sarda di Scienze Naturali 26: 305-310.

Bosc, G., Deschâtres, R. \& Paradis, G. (1992) Silene nocturna L. var. boullui (Jordan) Fiori \& Paol. In: Jeanmonod, D. \& Gamisans, J. (eds.), Notes et contributions à flore de Corse, VIII. Candollea 47: 288.

Briquet, J. (1910) Prodrome de la Flore Corse 1. Georg \& Co. Libraires Éditeurs, Genève, Bale, Lyon, 656 pp.

Brullo, C., Brullo, S., Giusso del Galdo, G., Ilardi, V. \& Sciandrello, S. (2012)A new species of Silene sect. Dipterosperma (Caryophyllaceae) from Sicily. Anales del Jardin Botánico de Madrid 69: 209-216. 
http://dx.doi.org/10.3989/ajbm.2327

Brullo, C., Brullo, S., Giusso, D.S., Minissale, P. \& Sciandrello, S. (2014) Silene peloritana (Caryophyllaceae) a new species from Sicily. Phytotaxa 172: 256-264.

http://dx.doi.org/10.11646/phytotaxa.172.3.6

Cafferty, S. \& Jarvis, C.E. (2004) Typification of Linnaean plant names in Caryophyllaceae. Taxon 53: 1049-1054. http://dx.doi.org/10.2307/4135573

Domina, G., Giusso Del Galdo, G., Gargano, D., Labra, M., Peccenini, S., Peruzzi, L. \& Raimondo F.M. (2012) The Italian Loci Classici Census. Taxon 61: 1351-1353.

Dutartre, G. (1987) Silene nocturna L. var. boullui (Jordan) Fiori \& Paol. In: Jeanmonod, D. \& Gamisans, J. (Eds.) Notes et contributions à flore de Corse, II. Candollea 42: 59.

Dutartre, G., Deschatres, L., Gamisans, J. \& Muracciole, M. (1986) Silene nocturna L. var. boullui (Jordan) Fiori \& Paol. In: Jeanmonod, D. \& Gamisans, J. (Eds.) Notes et contributions à flore de Corse, I. Candollea 41: 31-32.

Fawzi, N.M., Fawzy, A.M. \& Mohamed, A.A.-H.A. (2010) Seed morphological studies on some species of Silene L. (Caryophyllaceae). International Journal of Botany 6: 287-292.

http://dx.doi.org/10.3923/ijb.2010.287.292

Foggi, B., Grigioni, A. \& Luzzi, P. (2001) La flora vascolare dell'Isola di Capraia (Arcipelago Toscano): aggiornamento, aspetti fitogeografici e di conservazione. Parlatorea 5: 5-53.

Gamisans, J. \& Jeanmonod, D. (1993) Catalogue des plantes vasculaires de la Corse Ed. 2. Conservatoire et Jardin botaniques de la Ville de Geneve, Geneve, 258 pp.

Ghafoor, A. (1978) Caryophyllaceae. In: Jafri, S.M.H. \& El-Gadi, A. (Eds) Flora of Lybia 59. Al Faateh University, Tripoli, 122 pp.

Jeanmonod, D. (2007) Caryophyllaceae. In: Jeanmonod, D. \& Gamisans, J. (Eds.) Flora Corsica. Édisud, Aix-en-Provence, pp. 336360.

Jeanmonod, D. (2013) Caryophyllaceae. In: Jeanmonod, D. \& Gamisans, J. (Eds.) Flora Corsica. Société Botanique du Centre-Ouest, pp. 340-361.

Linnaeus, C. (1753) Species Plantarum 2. Laurentii Salvii, Stockholm, 639 pp.

Linnaeus, C. (1756) Centuria II Plantarum. Reg. Acad. Typogr., Uppsala, 38 pp.

Peruzzi, L. \& Carta, A. (2013) A taxonomic revision of Silene nocturna species complex (Caryophyllaceae) in Italy. Phytotaxa 88: $38-48$.

Peruzzi, L., \& Eroğlu, E. (2013) Karyotype asymmetry: again, how to measure and what to measure? Comparative Cytogenetics 7: 1-9. http://dx.doi.org/10.3897/compcytogen.v7i1.4431

Peruzzi, L., Jarvis, C.E. \& Carta, A. (2014a) On the application of the Linnaean names Cucubalus reflexus, Silene nocturna and Silene mutabilis (Caryophyllaceae). Taxon 63: 651-652.

http://dx.doi.org/10.12705/633.6

Peruzzi, L., Conti, F. \& Bartolucci, F. (2014b) An inventory of vascular plants endemic to Italy. Phytotaxa 168: 1-75.

Pinna, M.S., Fois, M., Fenu, G., Cañadas, E.M. \& Bacchetta, G. (2014) Silene valsecchiae Bocchieri. Informatore Botanico Italiano 45: 388-390.

Rouy, G. \& Foucaud, J. (1896) Flore de France 3. Société des sciences naturelles de la Charente Inférieure, 382 pp. http://dx.doi.org/10.5962/bhl.title.29592

Siljak-Yakovlev, S. \& Peruzzi, L. (2012) Cytogenetic characterization of endemics: past and future. Plant Biosystems 146: 694-702.

Sommier, S. (1898) Aggiunte alla florula dell'Isola di Capraia. Nuovo Giornale Botanico Italiano, nuova serie 5: 106-139.

Tenore, M. (1826) Ad Florae Neapolitanae Prodromum appendix quinta. Marotta \& Vanspandoch, Napoli, 34 pp.

Thiers, B. (2014) Index Herbariorum: A global directory of public herbaria and associated staff. New York Botanical Garden's Virtual Herbarium. Available from http://sweetgum.nybg.org/ih/ (accessed: 23 February 2014). 